Bundesgesundheitsbl 2013 · 56:1702-1705

DOI 10.1007/s00103-013-1864-5

(C) Springer-Verlag Berlin Heidelberg 2013

Bekanntmachung des Robert Koch-Institutes

\title{
Vorwort zur Liste der vom Robert Koch-Institut geprüften und anerkannten Desinfektionsmittel und -verfahren
}

ne/Desinfektionsmittel/desinfektionsmittel_node.html) veröffentlicht.

Alternierend zur Veröffentlichung einer zusammenfassenden Ausgabe der Liste aller geprüften und anerkannten Desinfektionsmittel und -verfahren im Bundesgesundheitsblatt, die an die Stelle der jeweils vorherigen tritt, werden Mittel und Verfahren, die zwischen 2 Ausgaben in die Liste aufgenommen wurden, jeweils in einem Nachtrag publiziert (weitere Einzelheiten unter oben genanntem Link).

Anträge zur Aufnahme von Desinfektionsmitteln und -verfahren in die Liste sind vom Hersteller beim Robert KochInstitut, Nordufer 20, 13353 Berlin, zu stellen; die dazu nötigen Formulare und Hinweise für Antragsteller finden sich ebenfalls unter oben genanntem Link auf den Internetseiten des RKI.

Bei Desinfektionsmitteln, die im medizinischen Bereich am menschlichen Körper angewendet werden sollen (z. B. Händedesinfektionsmittel), ist zu beachten, dass diese Präparate als Arzneimittel nach $₫ 2$ Abs. 1 des Arzneimittelgesetzes in Deutschland nur in den Verkehr gebracht werden dürfen, nachdem sie das BfArM zugelassen hat. Informationen zur Arzneimittelzulassung sind beim Bundesinstitut für Arzneimittel und Medizinprodukte, Kurt-Georg-Kiesinger-Allee 3, 53175 Bonn (http://www.bfarm.de) erhältlich.

Mittel zur Instrumentendesinfektion unterliegen als Zubehör zu Medizinprodukten in der Regel dem Medizinproduktegesetz. Dieses sieht für derartige Produkte eine $\mathrm{CE}-$ Kennzeichnung vor. Ab- grenzungsregelungen zu Biozidprodukten sind zu beachten (s. http://ec.europa. eu/health/medical-devices/files/wg_minutes_member_lists/borderline_manual_ol_en.pdf).

Desinfektionsmittel, die nicht zur Anwendung am menschlichen Körper bestimmt sind (z. B. Flächen- und Wäschedesinfektionsmittel) und die kein Zubehör zu Medizinprodukten sind, unterliegen der Biozid-Verordnung (EU Nr. 528/2012). Die Bundesanstalt für Arbeitsschutz und Arbeitsmedizin, Postfach 170202, 44061 Dortmund (http:// www.baua.bund.de) ist die deutsche $\mathrm{Zu}$ lassungsbehörde für Biozide.

Zur Prüfung der Wirksamkeit der Desinfektionsmittel bzw. -verfahren werden Methoden eingesetzt, die die vorgesehene Anwendungspraxis weitgehend berücksichtigen. Die Einzelheiten der Prüfmethodik sind in den Prüfrichtlinien des RKI festgelegt [1, 2, 3, 4, 5, 6, 13]. Für thermische Desinfektionsverfahren müssen zusätzlich die entsprechenden DINNormen herangezogen werden [18]. Mittel und Verfahren, für deren Anwendungsbereiche keine RKI-Testmethode beschrieben ist, z. B. Wäschedesinfektionsverfahren, werden mit den weiteren unter Prüfmethoden $[16,17,19,20]$ angegebenen Tests untersucht, wobei jeweils eine erhebliche organische Belastung simuliert wird.

Am Anfang der Liste stehen bewusst die thermischen Verfahren. Diese besitzen die größere Sicherheit gegenüber den chemischen Mitteln bzw. Verfahren. Zur Inaktivierung der relativ thermostabilen Hepatitis-B-Viren sind Verfahren 
mit feuchter Wärme $\geq 90^{\circ} \mathrm{C}$ erforderlich. Bakterielle Sporen können vorrangig bei Temperaturen $>100^{\circ} \mathrm{C}$ inaktiviert werden. In der Regel sind hierfür Sterilisationsverfahren erforderlich. Der Wirkungsbereich C (Milzbrandsporen) wird bereits mit geringeren Temperaturen erreicht. Zur Beherrschung bioterroristischer Gefährdungslagen, d. h. der bewussten und insbesondere großflächigen Ausbringung von Krankheitserregern oder deren Toxinen in der Umwelt, sind jedoch weitergehende Maßnahmen erforderlich, zu denen sich das RKI in anderen Publikationen äußert. Bei der Auswahl der Verfahren sind die Art des Erregers und das zu erwartende Ausmaß der Kontamination zu berücksichtigen.

Für Abfälle, die gemäß IfSG $\$ \$ 16$ und $17 \mathrm{zu}$ desinfizieren sind, eignen sich aus Gründen der Wirksamkeit sowie der Toxizität bzw. Ökotoxizität nur thermische Verfahren. Hierbei ist zu beachten, dass dabei entstehendes Kondensat und die Abluft so nachbehandelt werden müssen, dass von ihnen keine Gefahren ausgehen können. Letzteres kann in Sterilisationsgeräten nicht generell vorausgesetzt werden.

Die RKI-Liste enthält mit dem Wirkungsbereich B für die Mehrzahl der eingetragenen Mittel und Verfahren auch Angaben über deren Eignung zur Inaktivierung von Viren. Der Nachweis der Wirksamkeit gegen ein definiertes breites Spektrum von Viren ist die Voraussetzung für die Eintragung dieses Wirkungsbereiches in die RKI-Liste. Chemische Instrumentendesinfektionsmittel und nach dem März 1995 eingetragene Flächendesinfektionsmittel (mit * gekennzeichnet) wurden entsprechend der „Richtlinie des Robert Koch-Institutes zur Prüfung von chemischen Flächendesinfektionsmitteln und Instrumentendesinfektionsmitteln “ [6] in praxisnahen Untersuchungen getestet und für wirksam befunden. Die Eintragung des Wirkungsbereiches B für chemothermische Wäschedesinfektionsverfahren beruht auf den Ergebnissen von speziellen Suspensionsversuchen gemäß der Leitlinie der Deutschen Vereinigung zur Bekämpfung der Viruskrankheiten (DVV) e.V. und des Robert Koch-Instituts zur Prüfung von chemischen Desinfektions- mitteln auf Wirksamkeit gegen Viren in der Humanmedizin mit thermoresistenten bovinen (BPV) bzw. murinen (MVM) Parvoviren [13]. Eine Eintragung für den Wirkungsbereich B kann hierfür nur erfolgen, wenn für die vorgesehenen Anwendungsbedingungen auch die Wirksamkeit für den Wirkungsbereich A nachgewiesen worden ist.

Erstmals wird in der 16. Ausgabe der Liste bei Händedesinfektionsmitteln zusätzlich der Wirkungsbereich „,begrenzt viruzid“ aufgeführt. Die Eintragung erfolgt auf der Grundlage des Nachweises der Wirksamkeit durch Gutachten gemäß der oben angegebenen Leitlinie der DVV und des RKI [13]. Die Ergebnisse der vorgelegten Gutachten wurden in Nachprüfungen bestätigt. Die eingetragene Einwirkzeit ist aus Gründen der praktischen Durchführung der Händedesinfektion unter Berücksichtigung der in den praxisnahen Prüfungen zur Bakterizidie ermittelten Einwirkzeit abgeleitet, unabhängig davon, ob in den Gutachten zur Wirksamkeit gegen Viren ggf. kürzere Einwirkzeiten auf der Basis des oben genannten Suspensionstests ermittelt wurden.

Ebenfalls neu in dieser Ausgabe ist die Aufnahme von Sonderverfahren zur Behandlung von HEPA-Filtern in Sicherheitswerkbänken durch Begasung mit Formaldehyd oder Wasserstoffperoxid als Wirkstoff. Hinweise zur Eignung solcher Verfahren für die Raumbegasung sind unter Ziffer $3.3 \mathrm{zu}$ finden.

Eine Anforderung aus dem Bereich des Katastrophenschutzes, Schutzanzüge auch nach Kontamination mit bakteriellen Sporen so zu desinfizieren, dass die Infektionsgefahr für den Träger beim Ablegen weitgehend verringert wird, führte zur Entwicklung einer entsprechenden Prüfmethodik [14].

Seit dem Erscheinen der Norm „DIN EN ISO 15883 Reinigungs- und Desinfektionsgeräte" wurden keine weiteren Verfahren unter der Ziffer 3.2 aufgenommen. Bei den bisher dort eingetragenen Verfahren wurde jeweils nur für das gelistete Programm die mikrobiologische Wirksamkeit nachgewiesen, jedoch nicht die Reinigungsleistung beurteilt. Anforderungen für die Aufbereitung von Medizinprodukten in Reinigungs- und Desin- fektionsgeräten enthält die entsprechende Norm DIN EN ISO 15883. Ausführliche Erläuterungen zu Unterschieden zwischen den Anforderungen der Norm und der früheren RKI-Prüfung beinhaltet eine Mitteilung des RKI [12].

Desinfektionsmittel-Dosiergeräte, die bisher im Anhang der Liste aufgeführt wurden, unterliegen keiner Prüfung durch das RKI. Anforderungen für den Bau und den Betrieb dieser Geräte wurden in einer gemeinsamen Empfehlung der Bundesanstalt für Materialforschung und -prüfung, des Robert Koch-Institutes und der Kommission für Krankenhaushygiene und Infektionsprävention festgelegt [11]. Gemäß diesen Kriterien werden nur dezentral installierte Geräte empfohlen. Eine wesentliche Voraussetzung für den ordnungsgemäßen Betrieb besteht in der sachgerechten Wartung der Geräte.

Vorsorglich wird darauf aufmerksam gemacht, dass in Deutschland für routinemäßige Desinfektionsmaßnahmen im humanmedizinischen Bereich von der Desinfektionsmittel-Kommission des Verbundes für Angewandte Hygiene (VAH) eine Liste der nach den „Standardmethoden der DGHM zur Prüfung chemischer Desinfektionsverfahren" geprüften und als wirksam befundenen Desinfektionsverfahren herausgegeben wird. Anfragen hierzu sind an die Geschäftsstelle der DesinfektionsmittelKommission des VAH, Hygiene-Institut der Universität, Sigmund-Freud-Str. 25, 53127 Bonn, http://www.vah-online.de, zu richten. Die Desinfektionsmittel-Liste des VAH erscheint im mhp-Verlag, Ostring 13, 65205 Wiesbaden, http://www. mhp-verlag.de.

Zwischen der DesinfektionsmittelListe des RKI und der Desinfektionsmittel-Liste des VAH bestehen insbesondere in den Angaben zur Flächenund Instrumentendesinfektion wesentliche Unterschiede. Die Ursachen liegen in den unterschiedlichen Aufgaben der Listen und dementsprechend in den unterschiedlichen Prüfmethoden und Bewertungskriterien begründet. Die Liste des VAH ist in erster Linie auf die routinemäßige Desinfektion ausgerichtet, die Liste des RKI vornehmlich auf die behördlich angeordnete Desinfektion. 
Die zugrunde liegenden Prüfmethoden unterscheiden sich in der Art der Prüfanschmutzung und der Prüfkörper sowie in der Auswahl der Testorganismen. Bei Prüfungen durch das RKI wird als Prüfanschmutzung der Testobjekte erregerhaltiges gerinnendes Blut verwendet. Außerdem sind die Mykobakterien aufgrund ihrer hohen Resistenz generell in die Prüfungen für den Wirkungsbereich A eingeschlossen. Dadurch ergeben sich für viele Wirkstoffgruppen in der Liste des RKI höhere Konzentrationsangaben und/oder längere Einwirkzeiten. Für die Desinfektion grob verunreinigter Flächen können selbst diese Konzentrationen nicht in jedem Fall ausreichend sein. Derartige Verunreinigungen müssen von der Fläche zunächst entfernt und gesondert desinfiziert bzw. sicher entsorgt werden (s. auch „Vollzugshilfe zur Entsorgung von Abfällen aus Einrichtungen des Gesundheitsdienstes“ der LAGA, s. http://www.rki.de/DE/Content/Infekt/Krankenhaushygiene/Kommission/Downloads/LAGA-Rili.pdf? blob=publicationFile).

Zertifikate über die Wirksamkeit von Desinfektionsmitteln für den humanmedizinischen Bereich gegenüber Viren werden auch vom Fachausschuss Virusdesinfektion der Deutschen Vereinigung zur Bekämpfung der Viruskrankheiten veröffentlicht (http://www.dvv-ev. de/lfachausschuesse/Virusdesinfektion/ erteilte\%20Zertifikate\%2012\%209\%2010. pdf).

Für Desinfektionsmaßnahmen im veterinärmedizinischen und im Lebensmittelbereich gibt die Deutsche Veterinärmedizinische Gesellschaft (DVG) entsprechende Listen heraus. Diese sind bei der Geschäftsstelle der DVG, FriedrichStr. 17, 35392 Gießen bzw. über das Internet (http://www.dvg.net) zu beziehen.

Für den Hersteller von Desinfektionsmitteln besteht keine Verpflichtung, seine Präparate in die DesinfektionsmittelListen eintragen $\mathrm{zu}$ lassen. Insbesondere ist die Eintragung in die Listen keine Voraussetzung für den Marktzugang in Deutschland. Dem Anwender von Desinfektionsmaßnahmen steht die Wahl des Desinfektionsmittels grundsätzlich frei, soweit es sich nicht um behördlich angeordnete Maßnahmen, d. h. Entseuchun- gen gemäß $\$ 18$ IfSG handelt. Es ist jedoch dringend zu empfehlen, auch bei routinemäßiger Desinfektion die oben genannten Listen zu Rate zu ziehen, da bei den dort aufgeführten Mitteln und Verfahren die mikrobiologische Wirksamkeit durch Sachverständigengutachten belegt und von unabhängigen Institutionen bestätigt wurde.

\section{Literatur}

\section{Veröffentlichungen des RKI zur Prüfmethodik}

1. o A (1980) Richtlinie des Bundesgesundheitsamtes zur Prüfung von thermischen Desinfektionsverfahren in Reinigungsautomaten. Bundesgesundheitsblatt 23:364-367

2. o A (1992) Kommentar zur Richtlinie des Bundesgesundheitsamtes zur Prüfung von thermischen Desinfektionsverfahren in Reinigungsautomaten Bundesgesundheitsblatt 35:536-537

3. o A (1993) Prüfung von Abfalldesinfektionsverfahren auf Wirksamkeit. Bundesgesundheitsblatt 36:158-160

4. o A (1994) Richtlinie des Bundesgesundheitsamtes zur Prüfung der Wirksamkeit von Flächendesinfektionsmitteln für die Desinfektion bei Tuberkulose. Bundesgesundheitsblatt 37:274-278

5. o A (1994) Richtlinie des Robert Koch-Institutes zur Prüfung der Wirksamkeit von Desinfektionsmitteln für die chemische Instrumentendesinfektion bei Tuberkulose. Bundesgesundheitsblatt 37:474-477

6. o A (1995) Richtlinie des Robert Koch-Institutes zur Prüfung der Viruzidie von chemischen Flächendesinfektionsmitteln und Instrumentendesinfektionsmitteln, die in die Liste gemäß §10 c des Bundes-Seuchengesetzes aufgenommen werden sollen. Bundesgesundheitsblatt 38:242

7. o A (1996) Desinfektion und Sterilisation von chirurgischen Instrumenten bei Verdacht auf Creutzfeldt-Jakob-Erkrankungen. Bundesgesundheitsblatt 39:282-283

8. Simon D, Pauli G (1998) Krankenversorgung und Instrumentensterilisation bei CJK-Patienten und CJK-Verdachtsfällen. Bundesgesundheitsblatt 41:279-285

9. o A (2002) Die Variante der Creutzfeldt-JakobKrankheit (vCJK). Bundesgesundheitsblatt 45:376-394

10. o A (2004) Prüfung und Deklaration der Wirksamkeit von Desinfektionsmitteln gegen Viren. Stellungnahme des Arbeitskreises Viruzidie beim Robert Koch-Institut (RKI) sowie des Fachausschusses "Virusdesinfektion" der Deutschen Vereinigung zur Bekämpfung der Viruskrankheiten (DVV) und der Desinfektionsmittelkommission der Deutschen Gesellschaft für Hygiene und Mikrobiologie (DGHM). Bundesgesundheitsblatt 47:62-66

11. o A (2004) Anforderungen an Gestaltung, Eigenschaften und Betrieb von dezentralen Desinfektionsmittel-Dosiergeräten. Richtlinie der Bundes anstalt für Materialforschung und -prüfung, des Robert Koch-Institutes und der Kommission für Krankenhaushygiene und Infektionsprävention. Bundesgesundheitsblatt 47:67-72
12. o A (2007) Mitteilung des Robert Koch-Instituts zur Aufnahme von Reinigungs- und Desinfektionsgeräten in die Liste der geprüften und anerkannten Desinfektionsmittel und -verfahren gemäß §18 IfSG. Bundesgesundheitsblatt 50:128-129

13. o A (2008) Leitlinie der Deutschen Vereinigung zur Bekämpfung der Viruskrankheiten (DVV) e.V. und des Robert Koch-Instituts zur Prüfung von chemischen Desinfektionsmitteln auf Wirksamkeit gegen Viren in der Humanmedizin. Bundesgesundheitsblatt 51:937-945

14. Lemmer K, Roder A, Nattermann H et al (2012) Desinfektion Persönlicher Schutzausrüstung, Forschung im Bevölkerungsschutz, Band 17. Bundesamt für Bevölkerungsschutz und Katastrophenhilfe

15. Reichenbacher D, Thanheiser M, Krüger D (2010) Aktueller Stand zur Raumdekontamination mit gasförmigem Wasserstoffperoxid. HygMed 35:204-208

\section{Weitere Prüfmethoden}

16. DIN EN 1500 Chemische Desinfektionsmittel und Antiseptika - Hygienische Händedesinfektion Prüfverfahren und Anforderungen (Phase 2/Stufe 2). Deutsche Fassung EN 1500:2013

17. E DIN EN 16616 Chemische Desinfektionsmittel und Antiseptika - Chemothermische Wäschedesinfektion - Prüfverfahren und Anforderungen (Phase 2, Stufe 2); Deutsche Fassung prEN 16616:2013

18. DIN 58949 Desinfektion - Dampf-Desinfektionsapparate - Teil 2 (2001) und 3 (2012)

19. Gebel J, Werner H-P, Kirsch-Altena A, Bansemir K (2002) Standardmethoden der DGHM zur Prüfung chemischer Desinfektionsverfahren. Stand 1.9.2001. mhp-Verlag, ISBN 3-88681-042-9 bzw. jeweils aktuelle Fassung

20. Desinfektionsmittel-Kommission der DGHM: Anforderungskatalog für die Aufnahme von chemischen Desinfektionsverfahren in die Desinfektionsmittel-Liste der DGHM, Stand 4. Februar 2002. mhp-Verlag 2002, ISBN 3-88681-050-X bzw. jeweils aktuelle Fassung

21. Deutsche Veterinärmedizinische Gesellschaft e.V. Richtlinien für die Prüfung von Desinfektionsverfahren und chemischen Desinfektionsmitteln der DVG (4. überarbeitete Aufl. 2007-2012) erhältlich bei: Deutsche Veterinärmedizinische Gesellschaft

\section{Weiterführende Literatur}

22. Bertram J, Mielke M, Beekes M et al (2004) Inaktivierung und Entfernung von Prionen bei der Aufbereitung von Medizinprodukten. Bundesgesundheitsblatt 47:36-40

23. Bräuniger S, Fischer I, Peters J, Timm H (1995) Untersuchungen zur Prüfung der viruziden Wirksamkeit von Desinfektionsmitteln für die chemische Instrumentendesinfektion. HygMed 20:255262

24. Bräuniger S, Peters J, Borchers U, Kao M (2000) Further studies on thermal resistance of bovine parvovirus against moist and dry heat. Int J Environ Health 203:71-75

25. Peters J, Bräuniger S, Fischer I (1994) Zur Temperaturstabilität des bovinen Parvovirus. Zbl Hyg 196:270-278 
26. Peters J, Bräuniger S, Fischer I (1995) Zur Prüfung der viruziden Wirksamkeit von Flächendesinfektionsmitteln. HygMed 20:20-28

27. Peters J, Bräuniger S (1997) Untersuchungen zur Prüfung der viruziden Wirksamkeit von Desinfektionsmitteln für die chemische Instrumentendesinfektion. HygMed 22:497-503

28. Rabenau HF, Schwebke I, Steinmann J et al (2012) Quantitative Prüfung der viruziden Wirksamkeit chemischer Desinfektionsmittel auf nicht-porösen Oberflächen. Hyg Med 37:78-85

29. Renner P, Peters J (1996) Zur Prüfung der tuberkuloziden Wirksamkeit von Mitteln für die chemische Instrumentendesinfektion: Erprobung der Richtlinie des Robert Koch-Institutes an einigen Wirkstoffen. HygMed 21:271-277

30. Renner P, Peters J (1998/99) Resistenz von Enterokokken gegenüber Hitze und chemischen Agenzien. Zbl Hyg Umweltmed 202:41-50

31. Schaal H, Schulze-Röbbecke R, Thanheiser M, Glück W (2009) Sterilisation von HEPA-Filtern in Sicherheitswerkbänken der Klasse 2 mittels Formaldehydbegasung. HygMed 34(7/8):282286

32. Schwebke I, Rabenau H (2013) Aktueller Stand zur Viruzidieprüfung - ein Überblick. HygMed 37(7/8):291-295

33. Spicher G, Peters J (1985) Eine Methode zur Kontamination von Testobjekten mit gerinnendem Blut. Zbl Bakt Hyg B 182:89-94

34. Spicher G, Peters J (1991) Wirksamkeit von Formaldehyd, Glutardialdehyd, Peressigsäure, Chloramin T (N-Chlor-4-toluolsulfonsäureamid), $\mathrm{m}$ Kresol, Ethanol und Benzyldimethyldodecylammoniumbromid gegen Bakterien, die sich in geronnenem Blut befinden (Modellversuche zur chemischen Instrumentendesinfektion). Z $\mathrm{bl} \mathrm{Hyg}$ Umweltmed 191:457-477

35. Spicher G, Peters J (1997) Abhängigkeit der mikrobiologischen Befunde der Wirksamkeitsprüfung von Flächendesinfektionsmitteln von den Prüfungsbedingungen. HygMed 22:123-140

36. Spicher G, Peters J (1997/98) Beeinflussung der mikrobiziden Wirksamkeit von Formaldehyd, Glutardialdehyd, Peressigsäure, Chloramin T (NChlor-4-toluolsulfonsäureamid), m-Kresol, Ethanol und Benzyldimethyldodecylammoniumbromid durch Blut (Modellversuche zur chemischen Instrumentendesinfektion). Zbl Hyg Umweltmed 200:465-477 